\title{
Reactive Coordinated Optimal Operation of Distributed Wind Generation
}

\author{
Yue Xianga ${ }^{\text {, Lili Zhou }}{ }^{\mathrm{a}}$, Yuan Huang ${ }^{\mathrm{a}^{*}}$, Xin Zhang ${ }^{\mathrm{b}}$, Youbo Liu ${ }^{\text {a }}$, Junyong Liu ${ }^{\mathrm{a}}$ \\ ${ }^{a}$ College of Electrical Engineering, Sichuan University, Chengdu 610065, China \\ ${ }^{b}$ Energy and Power Theme, Cranfield University, Bedfordshire, MK43 OAL, United Kingdom
}

\begin{abstract}
Large-scale distributed wind generation (DWG) integration brings new challenges to the optimal operation of the distribution network. The reactive supports from wind turbines (WTs) and reactive power resources can improve both the operation economy and renewable energy consumption. In this paper, a multi-period reactive coordinated optimal operation model for DWG in the distribution network is established. The active-reactive power coordination characteristics of two typical types of WTs are considered and the operating strategy of reactive power resources is integrated in the model. The second-order cone programming (SOCP) is developed to transform the original nonlinear power flow model into a linear and convex model, which would significantly improve the power flow calculation efficiency for DWG penetrated distribution network. The simulation results show that the integration of reactive power resources can further promote the consumption of DWG and improve the operating profits of the distribution network.
\end{abstract}

Keywords distributed wind generation; optimal operation; active-reactive power coordination; reactive power resources; economic benefits

NOMENCLATURE

\begin{tabular}{|l|l|l|l|}
\hline \multicolumn{2}{|l|}{ Acronyms } & $\underline{\varphi}_{g, t}^{\text {grid }}, \bar{\varphi}_{g, t}^{\text {grid }}$ & $\begin{array}{l}\text { Lower/upper constraint limit of power } \\
\text { factor interacted with grid }\end{array}$ \\
\hline DWG & Distributed wind generation & $T$ & Typical dispatch period in a day \\
\hline WT & Wind Turbine & $\rho_{w}^{\text {Wom }}$ & Unit maintenance cost of DWG \\
\hline SOCP & Second-order cone programming & $\mathrm{M}$ & Big constant \\
\hline DFIG & Doubly fed induction generator & Variables \\
\hline FSWG & Fixed speed wind generator & $P_{w, t}^{D W G} / C_{w, t}^{D W G}$ & $\begin{array}{l}\text { Real time and forecast output of DFIG of } \\
\text { bus } w \text {-th at time } t\end{array}$ \\
\hline SCB & Shunt capacitor bank & $x_{w}^{D W G}$ & Installation status of DFIG of bus $w$-th. \\
\hline SVC & Static var compensator & $Q_{w, t}^{F S W G}$ & $\begin{array}{l}\text { Reactive power of FSWG of bus } w \text {-th at } \\
\text { time } t\end{array}$ \\
\hline Indices and sets & $P_{w, t}^{F S W G}$ & $\begin{array}{l}\text { Active power of FSWG of bus } w \text {-th at } \\
\text { time } t\end{array}$ \\
\hline$t / w$ & Indices for time/DWG & $\rho_{t}^{\text {loss }}$ & Power loss at time $t$ \\
\hline$i$ & Indices for buses & $\rho_{t}^{D W G}$ & Electricity price of DWG at time $t$ \\
\hline$i j$ & Indices for branches & $\rho_{t}^{\text {grid }}$ & Electricity price of grid purchase \\
\hline
\end{tabular}

*Corresponding author: yuanhuang@scu.edu.cn 


\begin{tabular}{|l|l|l|l|}
\hline$\Re$ & Set of decision variables & $P_{i j . t}, Q_{i j . t}$ & $\begin{array}{l}\text { Active and reactive power flow of } i j \text {-th } \\
\text { branch at time } t\end{array}$ \\
\hline$U S$ & Set of output uncertainty of DWG & $Q_{i, t}^{S C B}$ & $\begin{array}{l}\text { Reactive power output of SCB of } i \text {-th bus } \\
\text { at time } t\end{array}$ \\
\hline$\Omega_{D W G}$ & Set of DWG's candidate location & $Q_{i, t}^{S V C}$ & $\begin{array}{l}\text { Reactive power output of SVC of } i \text {-th } \\
\text { bus at time } t\end{array}$ \\
\hline$\psi(i)$ & $\begin{array}{l}\text { Set of branch end buses with bus } i \text { as the } \\
\text { head bus }\end{array}$ & $P_{i, t}^{L \text { Lad }} / Q_{i, t}^{\text {Load }}$ & Active/reactive load of $i$-th bus at time $t$ \\
\hline$\phi(i)$ & $\begin{array}{l}\text { Set of branch head point buses with bus } i \\
\text { as the end bus }\end{array}$ & $\tilde{V}_{i, t}, \tilde{I}_{i, t}$ & $\begin{array}{l}\text { Square of the voltage/current of } i \text {-th bus } \\
\text { at time } t\end{array}$ \\
\hline Constants & & $V_{i, t} / I_{i, t}$ & Voltage/current of $i$-th bus at time $t$ \\
\hline$\underline{\omega}_{w}^{D W G}, \bar{\omega}_{w}^{D W G}$ & $\begin{array}{l}\text { Lower/upper installation capability limit } \\
\text { of DWG }\end{array}$ & $I_{y, \xi, w, t}^{a / b / c / d}$ & DWG output indicators ranged 0-1 \\
\hline $\bar{N}_{\xi, r}^{\text {SCB }}$ & Upper installation number of SCB & Decision variables \\
\hline$\underline{V}_{i, t} / \bar{V}_{i, t}$ & $\begin{array}{l}\text { Lower/upper voltage limit of } i \text {-th bus at } \\
\text { time } t\end{array}$ & $x_{w}^{D W G}$ & Installation state of $w$-th DWG \\
\hline$\underline{I}_{i, t} \bar{I}_{i, t}$ & $\begin{array}{l}\text { Lower/upper current limit of } i j \text { branch at } \\
\text { time } t\end{array}$ & $\bar{P}_{w}^{D W G}$ & $\begin{array}{l}\text { Upper installation capability of } w \text {-th } \\
\text { DWG }\end{array}$ \\
\hline$\underline{P}_{g, t}^{\text {grid }} / \bar{P}_{g, t}^{\text {grid }}$ & $\begin{array}{l}\text { Lower/upper amount of power allowed to } \\
\text { purchase from grid }\end{array}$ & $P_{w, t}^{D W G}$ & $\begin{array}{l}\text { Active power output of } w \text {-th DWG at } \\
\text { time } t\end{array}$ \\
\hline
\end{tabular}

\section{Introduction}

Over the past few years, low-carbon generating technologies have been utilized to shape renewable energy and become increasingly popular worldwide. The distributed wind generation (DWG), which is planning flexible and environmentally friendly, is encouraged to be integrated into the distribution network. However, the integration of DWG has changed the power flow and demand pattern in a local distribution network, resulting in thermal overload, voltage management, and overall system reliability constraints [1]. Consequently, to cope with high wind energy penetration, active generation management becomes one of the promising solutions for the effective control of DWG [2], to achieve optimal operation of the distribution network and higher consumption level of DWG.

The planning and operation of the DG penetrated distribution network have been studied using several different methods. In [3], artificial intelligence and analytic algorithm were used to economically plan the DWG, based on minimizing the active power loss of the distribution network. Particle swarm optimization (PSO) was applied to solve the voltage and reactive power optimal control for DWG penetrated distribution network [4]. In [5], coordinated DGTie planning was analyzed using a genetic algorithm (GA). The intelligent algorithm is simple and straightforward, but it takes long calculating time and easy to fall into the optimal local solution. The distribution system operator must consider the power flow equation constraint for the operation layer, and its essence is the optimal power flow model. 
Besides, the traditional intelligent algorithm, the original dual interior point method [6] is more commonly used in the numerical solution for the nonlinear programming model. However, due to the nonlinear characteristics of the nonconvex penalty function and the strong nonconvex relaxation constraint, the traditional numerical solution cannot guarantee the optimal global solution [7]. Therefore, the sophisticated techniques, including convex programming methods, are more desirable. For instance, in article [8], Farivar M and Low S H established a branch flow model based on Distflow [9] with two relaxation steps. In the second step (i.e., second-order corn relaxation (SOCR)), the authors provided that optimal power flow (OPF) is convex and can be solved efficiently. Meanwhile, the accuracy of SOCR was further proved in [10]. Since then, SOCP have been used in many studies for power flow calculation, where SOCP is applied to transfer a model into convex programming. In [7], a robust planning model of active distribution network was established, and the second-order corn (SOC) was applied to transform the original model into a mixedinteger SOC programming problem to solve the constraint effectively. In [11], a dynamic optimal power flow framework based on SOCP was proposed, and its feasibility is verified by three examples in the active distribution network. A three-phase active and reactive coordination dynamic optimization model was presented in [12] and converted to a mixed-integer second-order cone programming using the SOC technique. With higher penetration of DG in the active distribution network, article [13] the sufficient accuracy condition for SOCR was supplemented and analyzed, and four IEEE standard systems were utilized to relax the system constraints. Besides, multi-objective optimization has been conducted to achieve high network reliability and economic planning of distribution networks, such as the minimization of active power loss to reduce investment and maintenance costs. The maximization of DG consumption to improve the economic operation of the distribution network [14], and other planning objectives, such as complex timing characteristics are analyzed [15]. However, most of the studies above used a single active power flow model rather than considering the voltage behaviors in the distribution network.

Different types of wind turbines(WTs) have different impacts on the power flow and voltage stability of the distribution network, which affect the consumption level of DWG. Also, the voltage control characteristics of different WTs types are required to be investigated [16]. The squirrel-cage asynchronous and doubly-fed induction generator based wind turbines were compared in articles [17]-[18] and the conclusion that the doubly-fed induction generator is more suitable in the distribution network for voltage control was addressed. However, most research works used wind generation as a way to control the voltage without considering their reactive output characteristics in the distribution 
network planning. For traditional voltage control of distribution network [19], on-load tap changer (OLTC) and reactive power compensation devices (e.g., SVC and shunt capacitor) were widely used for reactive power regulation. Integrating the wind generation's voltage control characteristics with traditional reactive power devices has become a new trend to manage voltage stability in the distribution network [20].

The problem of power quality, such as voltage fluctuation, is becoming more severe with a high rate of DWG penetration. If the voltage deviation or power flow exceeds the operational limits, there is a high possibility of curtailing the output of DWG for the distribution network's security. However, this approach discourages DWG's consumption and low-carbon incentives. Therefore, the utilization of DWG's voltage control and the optimal scheduling of reactive power resources would help to improve the voltage stability of an active distribution network. The integration of reactive power compensations was considered in [12] to optimize the voltage profiles of the system, and the result showed that active control of reactive power compensation could promote the consumption of DG while ensuring voltage quality. In [21], an optimal reactive power compensation method was proposed for grid-interactive cascaded PV systems to improve system operation performance and enhance DG consumption. In [22], reactive power optimization was employed with considering the planning and economic operation of the DWG penetrated distribution network. In [23], an intelligent coordinated Volt/VAr optimization approach was proposed to maximize energy savings for an active distribution system with DG, where voltage regulators and capacitor banks were used as control devices for voltage and reactive power control. In [24], a novel two-stage dynamic reactive power dispatch strategy was proposed to solve the coordinated dispatch problem of on-load tap changer, capacitor banks, and DGs in the distribution network. Similarly, an optimal reactive power dispatch strategy was proposed to minimize the total electrical losses of a DFIG-based wind farm in [25]. A novel two-stage dynamic reactive power dispatch strategy was proposed in [26] with considering the reactive power adjustment potential of DG. However, most of the studies above didn't consider the active and reactive power coordination characteristics, including the coordination of active and reactive resources, and the active-reactive power coordination characteristics of WTs as well.

In the previous studies, single active power models were used in optimal distribution network planning, i.e., the active and reactive power characteristics were often separated. In the distribution network, the economic operation is closely related to the active control of both active and reactive power devices (e.g., DWG and reactive power compensation equipment). In particular, the active-reactive power coordination characteristics to improve the 
consumption of DWG and economic operation of the distribution network. In addition, the traditional power flow calculation method based on active-reactive power decoupling is inapplicable to the distribution network. Some intelligent algorithms cannot also provide sufficient calculation speed and even obtain a globally optimal solution. Taken the above constraints into account, this paper studies the reactive power resources capability to support the economic operation of the distribution network and the consumption of DWG. The study in this paper is based on the current condition in China, where the principle DWG investor is a local electrical company but not an individual owner, i.e., the investment of DWG and distribution network is from the same utility. Of course, there are a few utilities in China, e.g., electricity retail companies, investing DWG too, but the number is reasonably small and are not considered in the paper. The main contributions of this paper are as follows:

- A multi-period reactive coordinated optimal operation model for DWG penetrated distribution network is established, aiming to maximize the economic operation of the distribution network and the consumption of DWG.

- The active-reactive power coordination characteristic of WTs and the configuration of reactive power resources are considered in the model.

- The original optimization model can be transformed into a convex and linear model by using the second-order cone programming (SOCP) method, which can improve the computational efficiency of the model solution.

The remainder of the paper is organized as follows: In Section 2, the active and reactive power coordination model to address the output uncertainty of different types of WTs is introduced. In Section 3, the multi-period reactive coordinated optimal operation model for DWG penetrated distribution network is intended to analyze the DWG operating benefit, where the SOCP method is used. In Section 4, the solution method using the Big-M algorithm is proposed. The case study is conducted in an IEEE 33-bus test system in Section 5. Finally, conclusions are drawn in Section 6.

\section{Active-reactive power coordination of WTs}

Different types of WTs have different impacts on voltage stability in the distribution network. Two typical types of WTs generating technologies are considered in this paper, which is the doubly-fed induction generator (DFIG) and fixed speed wind generator (FSWG).

\subsection{Active-reactive power coordination characteristics of DFIG}


$\mathrm{n}$ this paper, an active-reactive power coordination model is used [27]. The mathematical model is demonstrated in equation (1)-(2), combined with the capability curve of DFIG presented in Fig.1, where the points $\left(\underline{Q}_{w}^{G}, 0\right)$, $\left(P_{w, 1}^{G}, Q_{w, 1}^{G}\right),\left(P_{w, 2}^{G}, Q_{w, 2}^{G}\right),\left(P_{w, 3}^{G}, Q_{w, 3}^{G}\right),\left(P_{w, 4}^{G}, Q_{w, 4}^{G}\right)$ and $\left(\bar{Q}_{w}^{G}, 0\right)$ are defined along with the stator and rotor current limitations. Then, the capability curve is linearized as follows:

$$
\begin{gathered}
0 \leq P_{w, t}^{D W G} \leq x_{w}^{D W G} C_{w, t}^{D W G} \bar{P}_{w}^{D W G}, \forall w \in \Omega_{D W G} \\
P_{w, t}^{D W G} \leq \frac{p_{w, 1}^{G}}{Q_{w, 1}^{G}-Q_{w}^{G}}\left(Q_{w, t}^{D W G}-\underline{Q}_{w}^{G}\right) \\
P_{w, t}^{D W G} \leq \frac{P_{w, 2}^{G}-P_{w, 1}^{G}}{Q_{w, 2}^{G}-Q_{w, 1}^{G}}\left(Q_{w, t}^{D W G}-Q_{w, 2}^{G}\right)+P_{w, 2}^{G} \\
\forall Q_{w, t}^{D W G} \in\left[Q_{w, 1}^{G}, Q_{w, 2}^{G}\right] \\
P_{w, t}^{D W G} \leq \frac{P_{w, 3}^{G}-P_{w, 2}^{G}}{Q_{w, 3}^{G}-Q_{w, 2}^{G}}\left(Q_{w, t}^{D W G}-Q_{w, 3}^{G}\right)+P_{w, 3}^{G} \\
\forall Q_{w, t}^{D W G} \in\left[Q_{w, 2}^{G}, Q_{w, 3}^{G}\right] \\
P_{w, t}^{D W G} \leq \frac{P_{w, 4}^{G}-P_{w, 3}^{G}}{Q_{w, 4}^{G}-Q_{w, 3}^{G}}\left(Q_{w, t}^{D W G}-Q_{w, 4}^{G}\right)+P_{w, 4}^{G} \\
\forall Q_{w, t}^{D W G} \in\left[Q_{w, 3}^{G}, Q_{w, 4}^{G}\right]
\end{gathered}
$$

where $P_{w, t}^{D W G}$ and $C_{w, t}^{D W G}$ denote the real-time and forecast output of DFIG of bus $w$-th at time $t . \quad x_{w}^{D W G}$ indicates the installation status of DFIG of bus $w$-th. The $\left(Q_{w}^{G}, 0\right),\left(P_{w, 1}^{G}, Q_{w, 1}^{G}\right),\left(P_{w, 2}^{G}, Q_{w, 2}^{G}\right),\left(P_{w, 3}^{G}, Q_{w, 3}^{G}\right), \quad\left(P_{w, 4}^{G}, Q_{w, 4}^{G}\right)$ and $\left(\bar{Q}_{w}^{G}, 0\right)$ are the feature points of DFIG of bus $w$-th. Equation (2a) - (2d) are used to piece-wise linearized the nonlinear of active-reactive coordination characteristics of DFIG. 


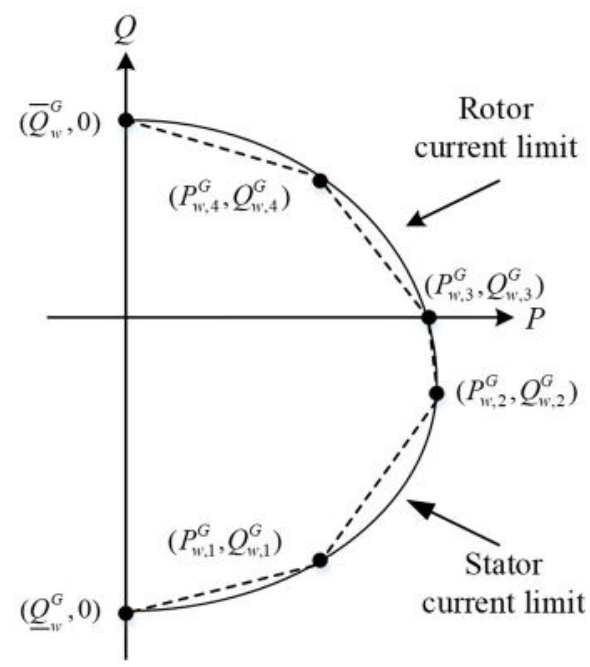

Fig.1 The capability curve of DFIG

\subsection{Active-reactive power coordination characteristics of FSWG}

A typically fixed speed wind generator (FSWG) is required to absorb reactive power in the distribution network, so voltage compensation equipment such as capacitors should be used to maintain the system voltage. Assuming that the power factor of FSWG is constant, the equation to represent the relationship between FSWG's active and reactive power output is shown in (3).

$$
Q_{w, t}^{F S W G}=0.95 \times P_{w, t}^{F S W G}
$$

where $Q_{w, t}^{F S W G}$ and $P_{w, t}^{F S W G}$ denote the reactive power and active power of FSWG.

\section{Multi-period reactive coordinated optimal operation model for DWG in distributed electricity network based on second-order cone programming (SOCP)}

The output of DWG can generate economic revenue for the DWG owner (e.g., distribution network), while network planners consider penetration levels when designing a low-carbon electricity network. Therefore, the maximum operation output and penetration level of DWG are considered as objective functions when planning the DWG in the distribution network. The timing characteristics of the distribution network with DWG are considered to maximize the profit of the distribution network.

\subsection{Objective function of DWG operation benefit}


The DWG scheduling period one day (24hours) is considered to maximize the operating benefit of DWG in the distribution network. Equation (4) demonstrates the proposed objective function, where the DWG's output and operation income, network's active power loss, and maintenance cost are considered.

$$
\begin{gathered}
\max _{P_{\xi, t}^{D W G} \in U S, x \in \Re} F=\sum_{t \in T}\left(\rho_{t}^{s u b}+\rho_{t}^{D W G}\right) \Delta t \sum_{w \in \Omega_{D W G}} P_{w, t}^{D W G}+\sum_{t \in T} \rho_{t}^{g r i d} \Delta t \sum_{w \in \Omega_{D W G}} P_{g, t}^{\text {grid }}- \\
\left(\sum_{t \in T} \rho_{t}^{\text {loss }} \Delta t \sum_{i j \in \Omega_{B}} r_{i j} \tilde{I}_{i j, t}+\sum_{w \in \Omega_{D W G}} \rho_{w}^{W o m} x_{w}^{D W G} \bar{P}_{w}^{D W G}\right)
\end{gathered}
$$

where $x$ denotes the decision variable. $\Re=\left\{x_{w}^{D W G}, \bar{P}_{w}^{D W G}, P_{w, t}^{D W G}\right\}$ denotes the set of the decision variables. $x_{w}^{D W G}$ and $\bar{P}_{w}^{D W G}$ represent the installation state and upper installation capability of $w$-th DWG. $P_{w, t}^{D W G}$ indicates the active power output of $w$-th DWG at time $t$. US is the set of output uncertainty of DWG. $\Omega_{D W G}$ is the set of DWG's candidate location. $T$ is the typical dispatch period in a day, which is 24 hours in this case. $\rho_{t}^{\text {loss }}, \rho_{t}^{\text {sub }}, \rho_{t}^{D W G}$ and $\rho_{t}^{\text {grid }}$ indicate the power loss, subsidy from government, electricity price $(\$ / \mathrm{MWh})$ of DWG and grid purchase. $\rho_{w}^{\text {Wom }}$ is the unit maintenance cost.

\subsection{Investment constraints}

$$
\begin{gathered}
\underline{\omega}_{w}^{D W G} \leq x_{w}^{D W G} \bar{P}_{w}^{D W G} \leq \bar{\omega}_{w}^{D W G}, \forall w \in \Omega_{D W G} \\
\sum_{w \in \Omega_{D W G}} x_{w}^{D W G} \leq X^{D W G}, \forall w \in \Omega_{D W G}
\end{gathered}
$$

where $\underline{\omega}_{w}^{D W G}$ and $\bar{\omega}_{w}^{D W G}$ denote the lower and upper installation capability limit of DWG. Equation (6) indicates the number of installation locations of DWG.

\subsection{Power flow constraints}

\section{(1) Traditional power flow model}

With the high penetration of DWG in the active distribution network, the direction of the power flow has changed from one way to multi-directional. However, similar to the traditional distribution network, the steady operation model is radially distributed. The optimal branch flow model based on distflow equations [8] is shown as (7)-(12).

$$
\begin{gathered}
s . t\left\{\begin{array}{l}
p_{j}=\sum_{k \in \delta(j)} P_{j k}-\sum_{i \in \pi(j)}\left(P_{i j}-I_{i j}^{2} r_{i j}\right)+g_{j} V_{j}^{2}, \forall j \in B \\
q_{j}=\sum_{k \in \delta(j)} Q_{j k}-\sum_{i \in \pi(j)}\left(Q_{i j}-I_{i j}^{2} x_{i j}\right)+b_{j} V_{j}^{2}, \forall j \in B
\end{array}\right. \\
V_{j}^{2}=V_{i}^{2}-2\left(P_{i j} r_{i j}+Q_{i j} x_{i j}\right)+I_{i j}^{2}\left(r_{i j}^{2}+x_{i j}^{2}\right), \forall i j \in E
\end{gathered}
$$




$$
\begin{gathered}
I_{i j}^{2}=\frac{P_{i j}^{2}+Q_{i j}^{2}}{V_{i}^{2}}, \forall i j \in E \\
\underline{I}_{i j} \leq I_{i j} \leq \bar{I}_{i j}, \forall i j \in E \\
\underline{V}_{j} \leq V_{j} \leq \bar{V}_{i}, \forall j \in B^{+} \\
\left\{\begin{array}{l}
p_{i} \in R_{j}^{p} \\
q_{j} \in R_{j}^{q}
\end{array}, \forall j \in B\right.
\end{gathered}
$$

(2) Relaxed power flow model

The traditional power flow is nonlinear, which increases the calculation complexity and leads to a sub-optimal computation speed. Therefore, a great incentive is presented to transfer the conventional power flow model into a linear model. An optional power flow framework for DWG penetrated distribution network is introduced with considering the reactive power compensator such as SCB and SVC. Equations (13)-(16) demonstrate the power flow model based on second-order cone programming (SOCP).

$$
\begin{aligned}
& \left(\sum_{j \in \psi(i)} P_{\xi, i j, t}-\sum_{k \in \phi(i)}\left(P_{\xi, i j, t}-\tilde{I}_{\xi, i j, t} r_{k i}\right)=\sum_{j \in \Omega_{D W G}} P_{\xi, i, t}^{D W G}-\sum_{j \in \Omega_{L o a d}} P_{\xi, i, t}^{L o a d}\right. \\
& \sum_{j \in \psi(i)} Q_{\xi, i j, t}-\sum_{k \in \phi(i)}\left(Q_{\xi, k i, t}-\tilde{I}_{\xi, k i, t} x_{k i}\right)=\sum_{i \in \Omega_{D W G}} Q_{\xi, i, t}^{D W G}+ \\
& \left\{\begin{array}{l}
\sum_{j \in \Omega_{S C B}} Q_{\xi, i, t}^{S C B}+\sum_{j \in \Omega_{\text {Load }}} Q_{\xi, i, t}^{\text {Load }}+\sum_{j \in \Omega_{S V C}} Q_{\xi, i, t}^{S V C} \\
\forall \xi \in \Omega_{\xi}, \forall i j \in \Omega_{B}, \forall t \in T
\end{array}\right. \\
& \left\{\begin{array}{l}
\tilde{V}_{\xi, j, t}=\tilde{V}_{\xi, j, t}-2\left(P_{\xi, i, t}+Q_{\xi, i j, t} x_{i j}\right)+\tilde{I}_{\xi, i j, t}\left(r_{i j}^{2}+x_{i j}^{2}\right) \\
\tilde{V}_{\xi, i, t} \tilde{I}_{i j, t}=P_{\xi, i j, t}^{2}+Q_{\xi, i j, t}^{2}, \\
\forall \xi \in \Omega_{\xi}, \forall i j \in \Omega_{B}, \forall t \in T
\end{array}\right. \\
& \left\{\begin{array}{l}
\left\|\begin{array}{l}
2 P_{\xi, i j, t} \\
2 Q_{\xi, i j, t} \\
\tilde{I}_{\xi, i j, t}-\tilde{V}_{\xi, i, t}
\end{array}\right\|_{2} \leq \tilde{I}_{\xi, i j, t}+\tilde{V}_{\xi, i, t} \\
\forall \xi \in \Omega_{\xi}, \forall i j \in \Omega_{B}, \forall t \in T
\end{array}\right. \\
& \left\{\begin{array}{l}
\tilde{V}_{\xi, i, t}=V_{\xi, i, t}^{2}, \tilde{I}_{\xi, i, t}=I_{\xi, i, t}^{2} \\
\forall \xi \in \Omega_{\xi}, \forall i j \in \Omega_{B}, \forall t \in T
\end{array}\right.
\end{aligned}
$$

Equation (13) represents the power balance with the constraint of Distflow. $\psi(i)$ denotes the collection of branch end bus with bus $i$ as the head bus. $\phi(i)$ denotes the set of branch head point buses with bus $i$ as the end bus. $\Omega_{\text {load }}$ is the set of load. $P_{i j . t}$ and $Q_{i j . t}$ indicate the active and reactive power flow of $i j$-th branch at time $t . Q_{i . t}^{S C B}$ and $Q_{i . t}^{S V C}$ are reactive power output of SCB and SVC of $i$-th bus at time $t . P_{i, t}^{L o a d}$ and $Q_{i, t}^{\text {Load }}$ denote the active and reactive load of $i$-th bus at time $t$. Equations (14) - (15) represent voltage constraints. $\tilde{V}_{i, t}$ is the square of the voltage of $i$-th bus at 
time $t . \quad \tilde{I}_{i, t}$ is the square of the current of $i$-th bus at time $t$. Equation (16) denotes the constraint of the second-order cone. $V_{i, t}$ and $I_{i, t}$ indicate the voltage and current of $i$-th bus at time $t$.

Equations (14) - (16) display the relaxed process to transform the original nonlinear model in equation (9) into a linear and convex model. A brief illustration of the SOCR process is depicted in Fig.2. In Fig. 2, $C_{\text {original }}$ represents a feasible set of the original model, which is nonlinear due to equation (9), $C_{s o c}$ represents the feasible convex set after SOCR, which contains $C_{\text {original }}$ obviously. The optimal solution $\mathrm{S}$ is a lower bound solution of the original model if $\mathrm{S}$ is a point in the set of $C_{\text {oruginal }}$. Thus, the condition constraint is satisfied.

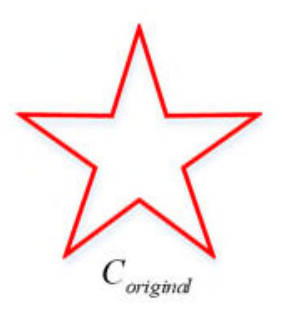

The original nonconvex feasible set

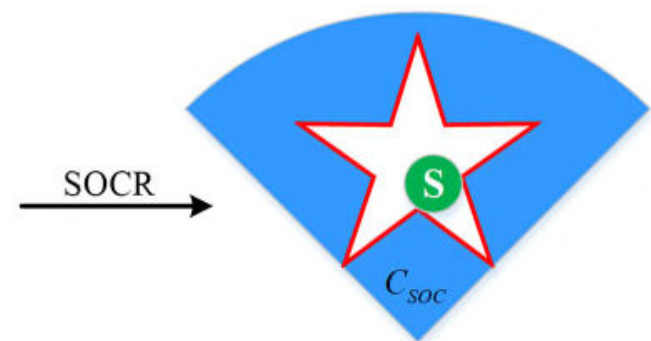

The convex feasible set after relaxation

Fig.2. The illustration of SOCR

\subsection{Shunt Capacitor Banks (SCBs) operating constraints}

$$
\begin{aligned}
& \sum_{t \in T} \delta_{\xi, r, t}^{\mathrm{SCB}} \leq \bar{N}_{\xi, r}^{\mathrm{SCB}} \\
& \left\{\begin{array}{l}
0 \leq Q_{\xi, r, t}^{S C B} \leq \bar{R}_{r}^{S C B} \\
\forall r \in \Omega_{S C B}, \forall t \in T
\end{array}\right.
\end{aligned}
$$

where equation (17a) limits the number of SCB. Equation (17b) is the constraint of SCB's output at each time.

\subsection{Static Var Compensator (SVC) operating constraints}

In order to reflect the universality of the method in this paper, that is, this paper can take into account the operating characteristics of the discrete-continuous reactive power compensation device, the SVC is taken into consideration.

Equation (18) denotes the operating constraint of SVC.

$$
Q_{\xi, t, \mathrm{SVC}}^{\min } \leq Q_{i, \mathrm{SVC}} \leq Q_{\xi, t, \mathrm{SVC}}^{\max }
$$

\subsection{Network operating state constraints}




$$
\begin{aligned}
\underline{V}_{\xi, i, t} & \leq V_{\xi, i, t} \leq \bar{V}_{\xi, i, t}, \forall \xi, \forall i, \forall t \\
\underline{I}_{\xi, i j, t} & \leq I_{\xi, i j, t} \leq \bar{I}_{\xi, i j, t}, \forall(i j) \in \Omega_{\mathrm{B}} \\
P_{\xi, g, t}^{\text {grid }} & \leq P_{\xi, g, t}^{\text {grid }} \leq \bar{P}_{\xi, g, t}^{\text {grid }}, \forall g \in \Omega_{\mathrm{G}} \\
\underline{\varphi}_{\xi, g, t}^{\text {grid }} & \leq \varphi_{\xi, g, t}^{\text {grid }} \leq \bar{\varphi}_{\xi, g, t}^{\text {grid }}, \forall g \in \Omega_{\mathrm{G}}
\end{aligned}
$$

Equation (19) denotes the nodal voltage constraint, where $\underline{V}_{i, t}$ and $\bar{V}_{i, t}$ indicate the lower and upper voltage limit of $i$ th bus at time $t$. Equation (20) denotes the branch current constraint, where $\underline{I}_{i, t}$ and $\bar{I}_{i, t}$ show the lower and upper current limit of $i j$ branch at time $t$. Equation (21) represents the constraint of power purchased from the grid. $\underline{P}_{g, t}^{\text {grid }}$ and $\bar{P}_{g, t}^{\text {grid }}$ are the most economical and highest amount of power allowed to purchase from the grid. Equation (22) is the constraint of the power factor interacted with grid. $\underline{\varphi}_{g, t}^{\text {grid }}$ and $\bar{\varphi}_{g, t}^{\text {grid }}$ indicate the lower and upper constraint limit of the power factor associated with the grid.

\section{Solution methods}

The equation (1)-(2) shown in Section 2 cannot be directly used for numerical optimization calculations. Therefore, Big-M algorithm is presented in the following steps

Firstly, variables $Q_{y, \xi, w, t}^{a}, Q_{y, \xi, w, t}^{b}, Q_{y, \xi, w, t}^{c}, Q_{y, \xi, w, t}^{d}$ and $P_{y, \xi, w, t}^{a}, P_{y, \xi, w, t}^{b}, P_{y, \xi, w, t}^{c}, P_{y, \xi, w, t}^{d}$ are employed, which represent the reactive power variables and active power variables of equations (2a)-(2d). Then the integral variables $I_{y, \xi, w, t}^{a}$, $I_{y, \xi, w, t}^{b}, I_{y, \xi, w, t}^{c}, I_{y, \xi, w, t}^{d}$ with a range of $0-1$ are used as indicators of DWG when generating. Finally, corresponding equality constraints are shown in (23)-(25).

$$
\begin{gathered}
I_{y, \xi, w, t}^{a}+I_{y, \xi, w, t}^{b}+I_{y, \xi, w, t}^{c}+I_{y, \xi, w, t}^{d}=1 \\
P_{y, \xi, w, t}^{a}+P_{y, \xi, w, t}^{b}+P_{y, \xi, w, t}^{c}+P_{y, \xi, w, t}^{d}=P_{y, \xi, w, t}^{D F I G} \\
Q_{y, \xi, w, t}^{a}+Q_{y, \xi, w, t}^{b}+Q_{y, \xi, w, t}^{c}+Q_{y, \xi, w, t}^{d}=Q_{y, \xi, w, t}^{D F I G}
\end{gathered}
$$

Equation (23) represents that only one of the indicators can be 1, i.e., the value the DWG's output can only be satisfied within a specified time interval. Equations (24) and (25) represent the sum of the reactive power and active power in four sections is the real-time output of DWG. Then, the constrains of the indicator variable $I$ will be simplified as follows.

$$
0 \leq P_{y, \xi, w, t}^{a} \leq I_{y, \xi, w, t}^{a} \times C_{y, \xi, w, t}^{D F I I} \bar{P}_{y, w}^{D F I G}
$$




$$
I_{y, \xi, w, t}^{a} \times\left(\frac{Q_{y, w, 1}^{G}-\underline{Q}_{y, w}^{G}}{P_{y, w, 1}^{G}} P_{y, \xi, w, t}^{a}+\underline{Q}_{y, w}^{G}\right) \leq Q_{y, \xi, w, t}^{a} \leq I_{y, \xi, w, t}^{a} \times\left(\frac{Q_{y, w, 4}^{G}-\bar{Q}_{y, w}^{G}}{P_{y, w, 4}^{G}} P_{y, \xi, w, t}^{a}+\bar{Q}_{y, w}^{G}\right)
$$

Expression (26) represents the restrictions that can be satisfied when $I_{y, \xi, w, t}^{a}$ is 1 , if $I_{y, \xi, w, t}^{a}$ is 0 , the $P_{y, \xi, w, t}^{a}$ and $Q_{y, \xi, w, t}^{a}$ are also 0. However, in equation (27), there is a quadratic variable $I_{y, \xi, w, t}^{a} \times C_{y, \xi, w, t}^{D F I G} \bar{P}_{y, w}^{D F I G}$, which can not be used in numerical optimization calculation as well. Therefore, the Big-M algorithm should be used to convert it into a primary variable, as shown in (28).

$$
\begin{gathered}
-M \times\left(1-I_{y, \xi, w, t}^{a}\right)+C_{y, \xi, w, t}^{D F I G} \bar{P}_{y, w}^{D F I G} \leq P I_{y, \xi, w, t}^{a} \leq M \times\left(1-I_{y, \xi, w, t}^{a}\right)+C_{y, \xi, w, t}^{D F I G} \bar{P}_{y, \xi, w, t}^{D F I G} \\
-M \times I_{y, \xi, w, t}^{a} \leq P I_{y, \xi, w, t}^{a} \leq M \times I_{y, \xi, w, t}^{a}
\end{gathered}
$$

where $\mathrm{M}$ is a constant, other item variables can be derived in parallel.

Then the transformed SOCP optimization problem could be solved by reliable solvers like CPLEX [28] directly.

\section{Case study}

A modified IEEE 33-bus system is used to test the proposed reactive coordinated optimization model with SOCP. All the algorithms are performed on a $1.6 \mathrm{GHz}$ computer with $4.0 \mathrm{~GB}$ RAM, and the proposed method is programmed in Matlab R2016a, where the mixed-integer conic programming is solved by CPLEX 12.6.0.

In order to more precisely demonstrate the improvement of the SOCP calculation efficiency, the calculation time of a mixed-integer nonlinear nonconvex programming (MINNP) model solved by genetic algorithm (GA) is compared with the linearized convex model by CPLEX solver in this paper. The result is shown in Table 1, which confirms the effectiveness of SOCP based on calculation time.

Table 1 Calculation time comparison

\begin{tabular}{ccc}
\hline Model type & Solution algorithm & Calculation time \\
\hline The original MINNP model & GA & $>1 \mathrm{~h}$ \\
The linearized convex model & CPLEX & $50.44 \mathrm{~s}$ \\
\hline
\end{tabular}

\subsection{Test system description}

IEEE 33-bus system is used as the test system, and the detailed parameters of the lines and peak load are given in the Appendix. The topology of the test system is shown in Fig. 3, where SCBs are integrated to buses 4 and 29; SVC are integrated to bus 31 , DWGs are integrated to buses $14,24,30$, and 32 . The specific information of unit capacity, 
installation quantity, installation cost, and maintenance cost is illustrated in Table 2. The unit capacity of DWG and SCB is $0.1 \mathrm{MW}$ and $0.1 \mathrm{Mvar}$, respectively. The step size of the SCBs is 0.05 MVar with 10 shunt capacitors in each $\mathrm{SCB}$, the reactive power compensation interval of SVC is from $-0.1 \mathrm{Mvar}$ to $0.3 \mathrm{Mvar}$. We assume that the voltage limit of each bus is $0.95 \sim 1.05$ p.u., the thermal rating of the network branch is $380 \mathrm{~A}$, and the initial load of the system is $3.715+\mathrm{j} 2.33 \mathrm{MVA}$, the power factor range of the branch connected to the main network is $0.95 \sim 1$. Setting bus 1 as the balancing bus, whose initial voltage amplitude is 1.0 p.u. The electricity price of the typical scenario within a day and the purchase price [29] are shown in Figure 4.

Table 2 Related parameters of installed equipment

\begin{tabular}{cccccc}
\hline Equipment & Bus & $\begin{array}{c}\text { Maximum } \\
\text { number of } \\
\text { installation }\end{array}$ & $\begin{array}{c}\text { Unit } \\
\text { capability }\end{array}$ & $\begin{array}{c}\text { Installatio } \\
\mathrm{n} \text { cost }(\$)\end{array}$ & $\begin{array}{c}\text { Maintenance } \\
\text { cost }(\$)\end{array}$ \\
\hline \multirow{2}{*}{ DFIG } & 4 & 10 & $0.1 \mathrm{MW}$ & $82.7 \mathrm{w}$ & $2 \mathrm{w}$ \\
& 14 & 10 & $0.1 \mathrm{MW}$ & $100 \mathrm{w}$ & $2 \mathrm{w}$ \\
& 30 & 10 & $0.1 \mathrm{MW}$ & $82.7 \mathrm{w}$ & $2 \mathrm{w}$ \\
FSWG & 4 & 10 & $0.1 \mathrm{MW}$ & $82.7 \mathrm{w}$ & $2 \mathrm{w}$ \\
& 14 & 10 & $0.1 \mathrm{MW}$ & $100 \mathrm{w}$ & $2 \mathrm{w}$ \\
SCB & 30 & 10 & $0.1 \mathrm{MW}$ & $82.7 \mathrm{w}$ & $2 \mathrm{w}$ \\
& 4 & 10 & $0.01 \mathrm{Mvar}$ & $/$ & $/$ \\
SVC & 29 & 10 & $0.01 \mathrm{Mvar}$ & $/$ & $/$ \\
\hline
\end{tabular}




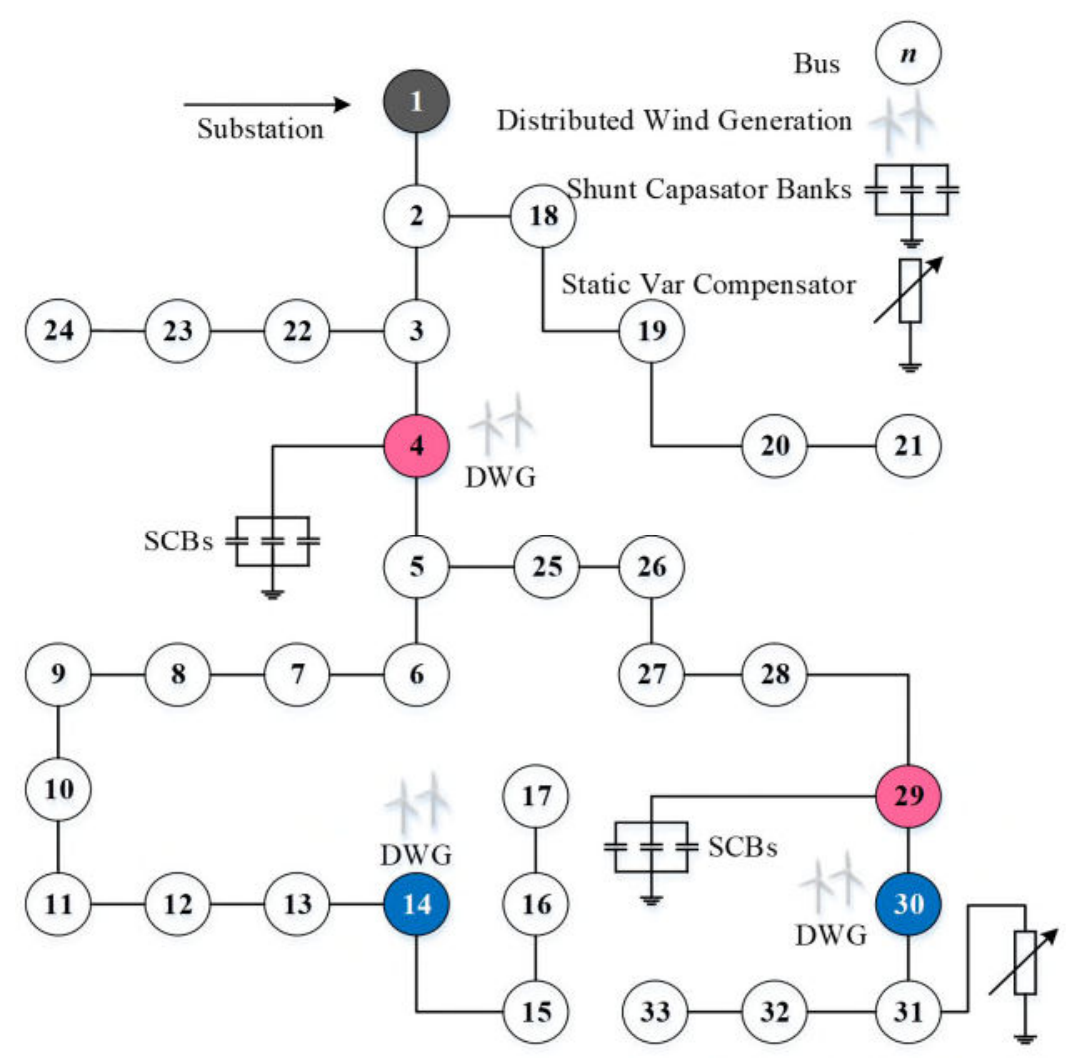

Fig. 3 Test System

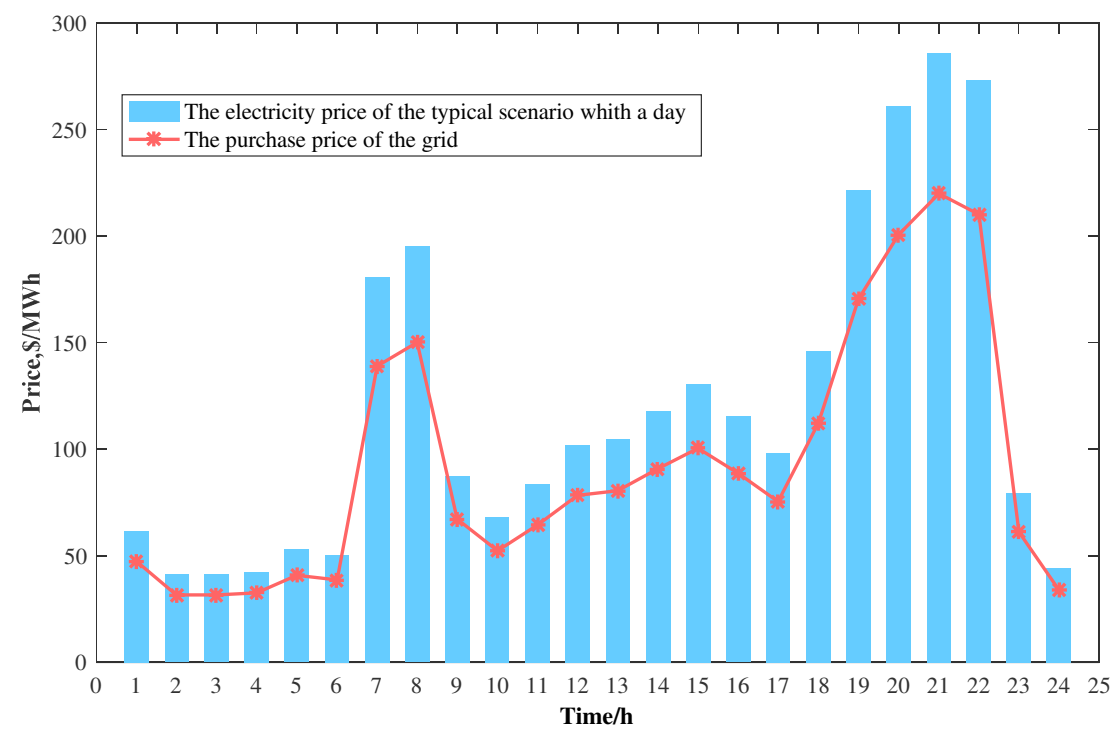

Fig.4 Electricity price

\subsection{Different types of WTs in the distribution network}

The voltage impact, DWG operation benefits, and power loss for DFIG and FSWG integration are shown and compared in Fig. 3 and Table 3, respectively. For the same location and installation capacity, DFIG generates more 
operation income than FSWG. In the meantime, due to the active and reactive power support that DFIG provides, the power flow drawn from the transmission network is significantly reduced, which leads to the lower power loss cost as well as less voltage drop. Also, the voltage deviation shows a smoother trend with DFIG for a continuous 24 hours, as shown in Fig.5., which improves voltage management throughout the schedule. Therefore, DFIG can be concluded as more effective than FSWG in terms of voltage management when being integrated into the distribution network.

Table 3 Results of different types of WTs integrated to the distribution network

\begin{tabular}{cccccc}
\hline WT Types & Bus & $\begin{array}{c}\text { Maximum } \\
\text { Installation } \\
\text { capability } \\
\text { (MW) }\end{array}$ & $\begin{array}{c}\text { distribution } \\
\text { network } \\
\text { operation } \\
\text { Income (\$) }\end{array}$ & $\begin{array}{c}\text { Power loss } \\
\text { cost } \$ \text { ) }\end{array}$ & $\begin{array}{c}\text { The average of } \\
\text { voltage drop\% } \\
\text { (p.u.) }\end{array}$ \\
\hline \multirow{2}{*}{ DFIG } & 4 & 1 & & & \\
& 14 & 1 & 147886.7 & 178.96 & 1.054 \\
& 30 & 1 & & & \\
FSWG & 4 & 1 & & & 1.098 \\
& 14 & 1 & 145598.8 & 204.03 & \\
\hline
\end{tabular}

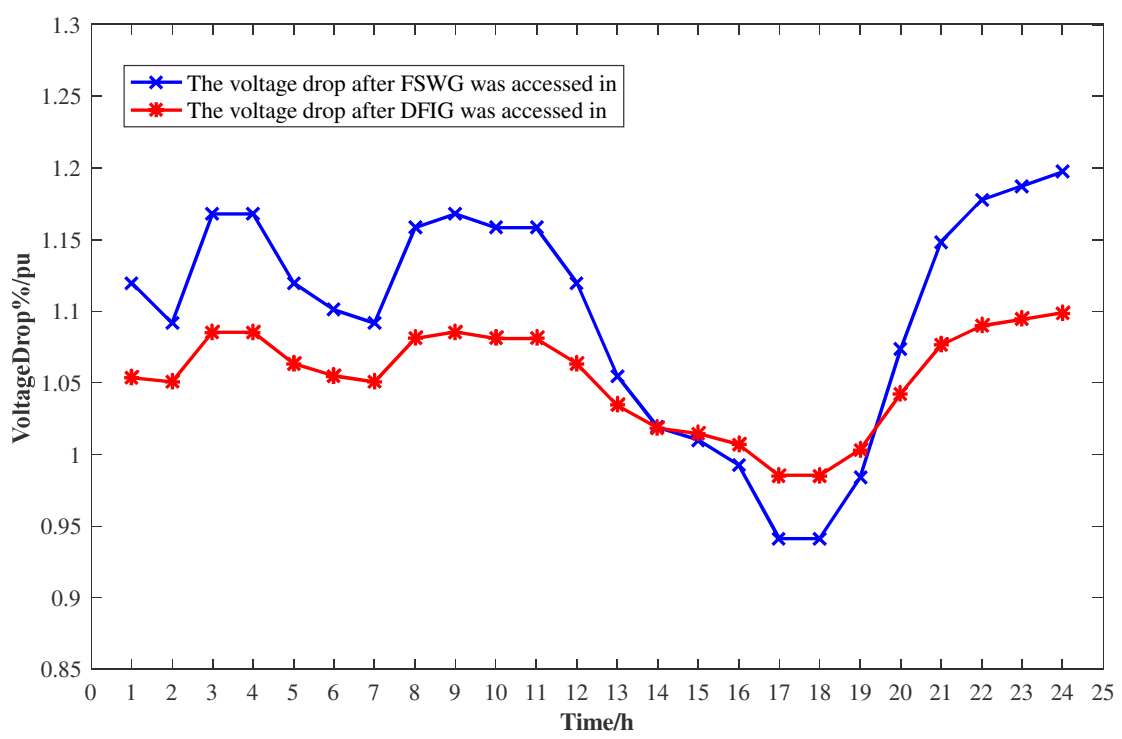

Fig. 5 Voltage drop with different types of WTs integration

In the above analysis, the voltage drop and economic impact are mainly discussed. Without considering the pre-set maximum install capacity limitation, the maximum DWG installation capacities with different types of WTs are given in Table 4.

Table 4 Maximum installation capacity with different types of WTs 


\begin{tabular}{cccc}
\hline WT types & Bus & $\begin{array}{c}\text { Maximum Install } \\
\text { capability (MW) }\end{array}$ & $\begin{array}{c}\text { Sum of maximum } \\
\text { installation capacity (MW) }\end{array}$ \\
\hline \multirow{2}{*}{ DFIG } & 4 & 2.6 & 5.5 \\
& 14 & 1.3 & \\
& 30 & 1.6 & 5.5 \\
FSWG & 4 & 1.0 & \\
& 14 & 3.2 & \\
\hline
\end{tabular}

As can be seen from Table 4 that the sum of maximum DWG installation capacities with different types of WTs is 5.5MW, but the installation capacities at different buses are different. When the WT type is DFIG, the installation capacity is more reasonable due to bus 4 closeness to the transformer. The larger DWG capacity can better respond to the system requirements. Comparing the DFIG installation buses 14 and 30, the load of bus $30(0.2+\mathrm{j} 0.6 \mathrm{MVA})$ is more significant than a load of bus $14(0.12+\mathrm{j} 0.08 \mathrm{MVA})$ required more DWG investment. Therefore, combining with previous conclusions, the results show that the DFIG is much suitable for integration to the distribution network in the case, compared with FSWG.

The following will further discuss the difference in the effect of the reactive power compensation device on different wind turbines and observe the comparison of the operating income and network loss of the distribution network with different capacity reactive power compensation accessed in as shown in Table 5.

Table 5 DFIGand FSWG operation results with different capacity of reactive power compensation device

\begin{tabular}{|c|c|c|c|}
\hline WTs types & $\begin{array}{c}\text { Reactive power } \\
\text { compensation device } \\
\text { capacity (Mvar) }\end{array}$ & $\begin{array}{l}\text { Distribution network } \\
\text { operation Income (\$) }\end{array}$ & Power loss cost $(\$)$ \\
\hline \multirow{3}{*}{ DFIG } & $0 \mathrm{SCB}+0 \mathrm{SVC}$ & 147886.7 & 178.96 \\
\hline & $\begin{array}{c}0.1 \mathrm{SCB}+ \\
{[-0.10 .3] \text { SVC }}\end{array}$ & 151509.4 & 139.2 \\
\hline & $0 \mathrm{SCB}+0 \mathrm{SVC}$ & 145598.8 & 204.03 \\
\hline FSWG & $\begin{array}{c}0.1 \mathrm{SCB}+ \\
{[-0.10 .3] \mathrm{SVC}}\end{array}$ & 151364.3 & 140.8 \\
\hline
\end{tabular}

It can be seen from the Table 5 that FSWG is more dependent on the coordination of reactive power compensation resources than DFIG. After the addition of reactive power compensation device, the operating efficiency of FSWG has been greatly improved, and the network loss has also been significantly reduced. Therefore, from the perspective of coordination effect, reactive resources have a better coordination effect on FSWG. 


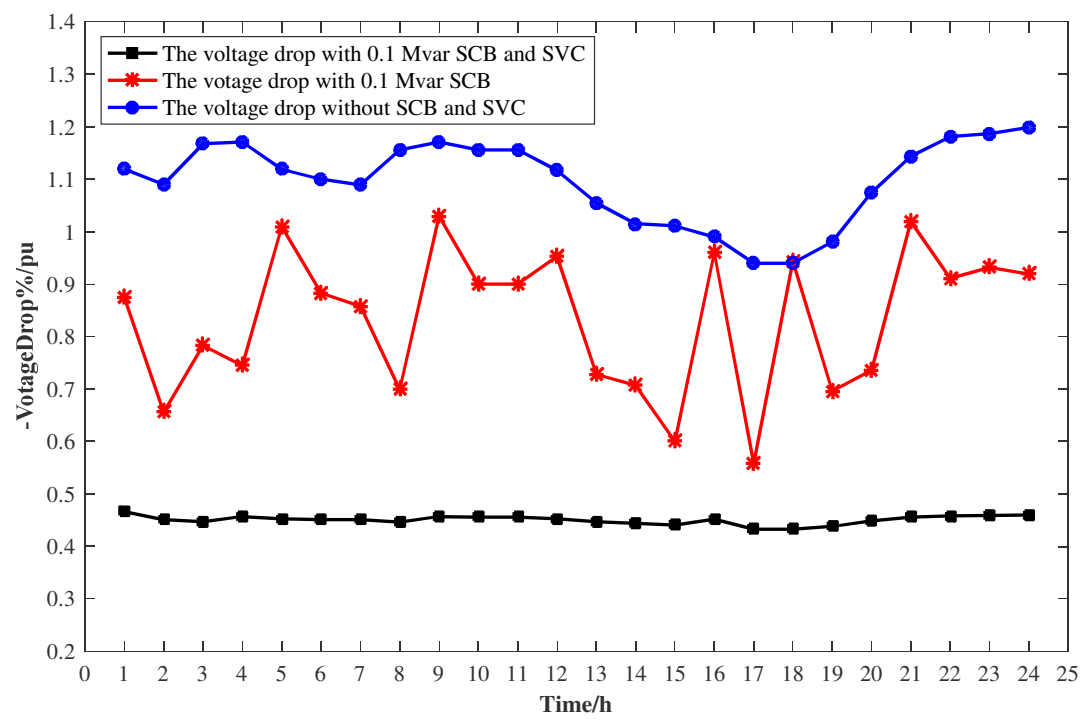

Fig. 6 Voltage drop within a day with different SCBs capacity

Reactive compensation devices can help active distribution network to achieve effective voltage drop control for DWG operation. The widely used SCB [30] and SVC are considered as the main reactive compensation devices in this paper. In order to analyze whether the coordination of reactive compensation device is beneficial to the distribution network voltage control, the voltage drop with $0.1 \mathrm{Mvar}$ SCB and [-0.1 0.3]Mvar SVC is compared with that with no SCB or with 0.1 Mvar SCB in a day, and the result is shown in Fig. 6.

It can be seen from the Fig.6 that the voltage performance of the distribution network could be improved by the coordination of SCBs and SVC, i.e., the coordination of reactive power compensation devices can further realize the friendly control of the DWG in the distribution network, thereby saving the power consumption of the DWGs.

Based on the optimal operation results of different WT types integrated into the distribution network shown in Section 5.2. DFIG is selected to achieve the desired operation income in this section. The changes in SCB capacity and DFIG operation income and power loss cost are listed in Table 6.

Table 6 DFIG operation result with different capacity of the reactive power compensation device

\begin{tabular}{cccc}
\hline $\begin{array}{c}\text { Reactive power } \\
\text { compensation device } \\
\text { capacity (Mvar) }\end{array}$ & $\begin{array}{c}\text { distribution network } \\
\text { operation Income (\$) }\end{array}$ & $\begin{array}{c}\text { Power loss } \\
\text { cost (\$) }\end{array}$ & $\begin{array}{c}\text { Install capability of } \\
\text { DFIG (MW) }\end{array}$ \\
\hline 0 SCB + 0 SVC & 147886.7 & 178.96 & 3 \\
0.1 SCB + 0 SVC & 150060.2 & 145.78 & 3
\end{tabular}




\begin{tabular}{cccc}
$0 \mathrm{SCB}+[-0.10 .3] \mathrm{SVC}$ & 151096.4 & 141.18 & 3 \\
$0.1 \mathrm{SCB}+[-0.10 .3] \mathrm{SVC}$ & 151509.4 & 139.3 & 3 \\
\hline
\end{tabular}

The installation of SCB and SVC certainly reduce the power loss cost, and SVC works better than SCB. At the same time, with the increase of SCB's capacity, the operating profit of the distribution network has also slightly improved. Then, the optimal output of DFIG with different capacities of SCB is presented in Fig. 7.

The main factor limiting the consumption of DWG is voltage stability, which is closely related to reactive power management. In the distribution network, the power lines appear to be more resistive characteristics $(\mathrm{R}>>\mathrm{X})$. The equation of voltage drop $\Delta V=\frac{P R+Q X}{V}$ shows that the impact of active power to voltage drop is more effective than reactive power. However, active power flow could not be reduced due to the requirement of meeting local demand. Therefore, the reduction of reactive power flow on the line is an alternative way to improve the system voltage, which can be achieved by using SCBs. Fig. 7 shows that the active power output of DFIG is developed to generate a large amount of operating revenue with the increasing of the SCB's capability, which demonstrates that reactive compensation devices can support the economic operation of the distribution network while improving the level of the consumption of DWG.

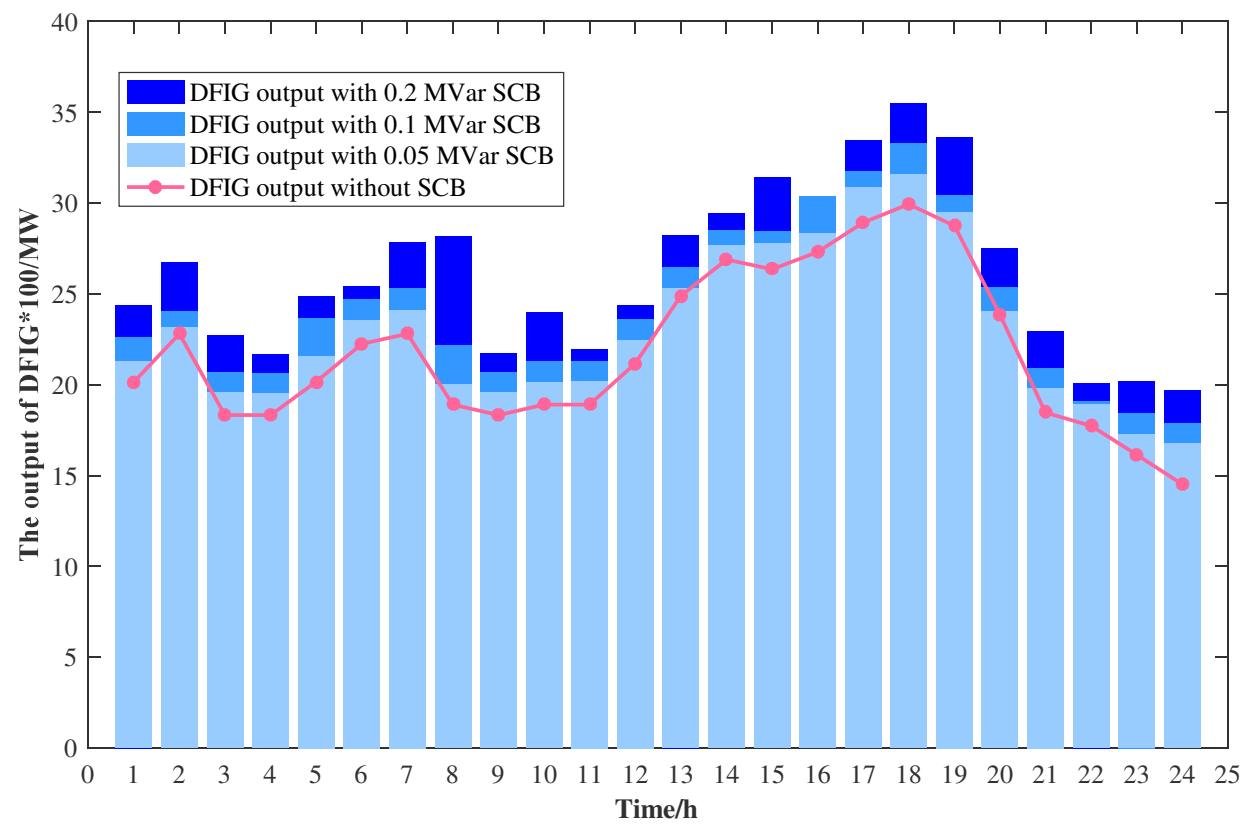

Fig. 7 Output of DFIG with different SCBs capacity

In the environment of large-scale DG access, when overvoltage occurs in the operation of the distribution network, 
the traditional control method is to curtail part of the DWG output to maintain the safe operation of the power grid, resulting in the abandonment of wind, which is contrary to the strategy of making full use of new energy to generate electricity. In the method proposed in this paper, the objective is optimized without limiting the DWG output, so that it can be concluded that the method in this paper can improve the ability of the grid to absorb DG. In order to further illustrate the promotion of reactive power compensation devices on DFIG consumption, the part of wind abandon rate of DFIG with and without reactive power compensation devices comparison is given in Table 7.

Table 7 Wind abandon rate of DFIG with and without reactive power compensation devices

\begin{tabular}{ccc}
\hline \multirow{2}{*}{ Time } & \multicolumn{2}{c}{ Wind abandon rate of DFIG } \\
\cline { 2 - 3 } & $\begin{array}{c}\text { Without SCB and } \\
\text { SVC/\% }\end{array}$ & $\begin{array}{c}\text { With 0.1Mvar SCB and } \\
{[-0.1}\end{array}$ \\
\hline & $0.3]$ Mvar SVC/\% \\
\hline 2 & 9.3 & 2.3 \\
6 & 9.2 & 3.2 \\
8 & 10 & 0 \\
10 & 10 & 0 \\
18 & 16.5 & 9 \\
24 & 15.3 & 0 \\
\hline
\end{tabular}

It can be seen from Table 7 that before the addition of the reactive power compensation device, DFIG has abandoned wind in multiple time periods. After the addition, the real-time abandonment rate of DFIG in each time period of the grid operation scheduling cycle is greatly reduced.

\section{Conclusion}

In this paper, a multi-period optimal operation model for DWG in the distribution network is established. The active and reactive power coordination characteristics of different types of WTs (DFIG and FSWG) are considered for the power output and voltage control model. The operational benefits of the DWG penetrated distribution network is analyzed through the proposed model with various technical and commercial constraints. The configuration of shunt capacitor banks is also studied to develop the optimal operation of the DWG penetrated distribution network. The second-order cone programming (SOCP) method is employed to enhance the calculation efficiency of the coordinated optimization model. Also, the case studies are performed in the IEEE 33-bus system. The main findings based on the case study include:

- The WT type directly affects the voltage and reactive power management of the distribution network. The 
simulation results demonstrate that the average voltage drop with DFIG integration is much lower than FSWG, and higher operating profit is perceived with DFIG integration. Consequently, DFIG is more effective in voltage control of the distribution network and has significant economic benefits, but reactive resources have a better coordination effect on FSWG.

- The integration of reactive power resources can further promote the consumption of DWG and enhance the operating income of the distribution networks. In active distribution network, the consumption of DWG is almost related to system voltage stability. The SCBs and SVC could reduce the reactive power flow on the distribution network, and minimize the voltage drop caused by DWG penetration. Such reactive compensation devices can increase the overall output of DWG during continuous wind energy scheduling.

- The nonconvex and nonlinear model of the distribution network can be transferred to a convex and linear model by SOCP, which can improve the calculation efficiency of the DWG penetrated distribution network.

Based on the proposed method, the improved model of DWGs' operation considering different owners and coordination of energy storage resources will be investigated in the future.

\section{Acknowledgment}

This work was supported in part by the Young Scholar Support Program of the Chinese Society of Electrical Engineering (CSEE-YESS-2018006), in part by the Scientific Foundation for the National Natural Science Foundation of China (51807127).

\section{References}

[1] He Jinghan, Liu Lin, Ding Fanfan, Li Changcheng, Zhang Dahai. A new coordinated backup protection scheme for distribution network containing distributed generation. Protect Control of Modern Power System, 2(2), pp:2-10, 2017.

[2] Zhiwei Cui, Xiaoqing Bai, Peijie Li, et al. "Optimal Strategies for Distribution Network Reconfiguration Considering Uncertain Wind Power,” CSEE Journal of Power and Energy System, 6(3), pp:662-671, 2020.

[3] Mahmoud K, Yorino N, Ahmed A, "Optimal distributed generation allocation in distribution networks for loss minimization,” IEEE Trans. Power Syst., vol.31, no.2, pp:1-10, 2015. 
[4] Rahul Anilkumar, Griet Devriese and Anurag K. Srivastava. "Voltage and reactive power control to maximize the energy savings in power distribution system with wind energy," IEEE Trans. industry applications, vol.54, no.1, pp: 656-664, 2018.

[5] Y. Xiang, L. Zhou, Y. Su, et al., "Coordinated DG-Tie planning in distribution networks based on temporal scenarios," Energy, vol.159, pp:774-785, 2018.

[6] Zhang Shenxi, Li Ke, Cheng Haozhong, et al. "Siting and sizing planning of distributed WTs under active management mode,". Automation of Electric Power System, vol.39, no.9, pp: 208-214, 2015.

[7] H. Gao, J. Liu, L. Wang, "Robust coordinated optimization of active and reactive power in active distribution networks," IEEE Trans.Smart Grid., vol.9, no.5, pp:4436-4447, 2017.

[8] Farivar M, Low S H. "Branch flow model: relaxations and convexification-part I," IEEE Trans.Power Syst., vol.28, no.3, pp:2554-2564, 2013.

[9] Baran M E, Wu F F. "Network reconfiguration in distribution networks for loss reduction and load balancing," IEEE Trans. Power Syst., vol.4, no.2, pp:1401-1407, 1989.

[10] Farivar M, Low S H. "Branch flow model: relaxations and convexification-part II," IEEE Trans. Power Syst., vol.28, no.3, pp:2565-2572, 2013.

[11]H. Gao, J. Liu, X. Shen, et al., "Optimal power flow research in active distribution network and its application examples," Proceedings of the CSEE, vol.37, no.6, pp:1634-1644, 2017.

[12] Q. Guo, J. Wu, C. Mo, et al., “A model for multi-objective coordination optimization of voltage and reactive power in distribution networks based on mixed integer second-order cone programming," Proceedings of the CSEE, vol.38, no.5, pp:1385-1396, 2018.

[13] L. Gan, N. Li, Topcu U, et al., "Exact convex relaxation of optimal power flow in radial networks," IEEE Transactions on Automatic Control, vol.60, no.1, pp:72-87, 2015.

[14] Shaaban M F, Atwa Y M, El-Saadany E F, “DG allocation for benefit maximization in distribution networks,” IEEE Trans. Power Syst., vol.28, no.2, pp:639-649, 2013.

[15] Y. Gao, X. Hu, W. Yang, et al., "Multi-objective bilevel coordinated planning of distributed generation and distribution network frame based on multiscenario technique considering timing characteristics," IEEE Trans. Sustainable Energy, vol.8, no.4, pp:1415-1429, 2017.

[16] N. Cao, Y. Li, H. Zhao, et al., "Comparison of effect of different wind turbines on power grid transient stability," Power System Technology, vol.31, no.9, pp:53-57, 2007. 
[17] Andres E. Leon, Marcelo F. Farias, Pedro E. Battaiotto, et al., "Control strategy of a DVR to improve stability in wind farms using squirrel-cage induction generators,” IEEE Trans. Power Syst., vol.26, no.3, pp:1609-1617, 2011.

[18] Y. Lang, X. Zhang, D. Xu, et al., "Reactive power analysis and control of doubly fed induction generator wind farm," Proceedings of the CSEE, vol.27, no.9, pp:77-82, 2007.

[19] Mohammad Nazmul Islam, Lasantha Gunaruwan Meegahapola and Manoj Datta. "Reactive power management in renewable rich power grids: a review of grid-codes, renewable generators, support devices, control strategies," IEEE Access, vol.6, pp: 41458-41489, 2018.

[20] J. Liu, H. Gao, Z. Ma, and Y. Li, "Reactive and prospect of active distribution network planning", Modern Power Syst. Clean Energy, vol.3, no.4, pp: 457-467, 2015.

[21] L. Liu, H. Li, Y. Xue, W. Liu, "Reactive power compensation and optimization strategy for grid-interactive cascaded photovoltaic systems,” IEEE Transactions on Power Electronics, vol.30, no.1, pp:188-202, 2015.

[22] T. Ding, S. Liu, W. Yuan, et al., “A two-stage robust reactive power optimization considering uncertain wind power integration in active distribution networks," IEEE Transactions on Sustainable Energy, vol.7, no.1, pp: 301-311, 2016.

[23] Rahul Anilkumar, Griet Devriese and Anurag K. Srivastava. "Voltage and reactive power control to maximize the energy saving in power distribution system with wind energy," IEEE Transaction on Industry Application, vol.54, no.1, pp: 656-664, 2018.

[24] Sudipta Ghosh, Younes J. Isbeih, Rojan Bhattarai, et al. "A dynamic coordination control architecture for reactive power capability enhancement of the DFIG-based wind power generation," IEEE Transaction on Power System, vol.35, no.4, pp: 3051-3064, 2020.

[25] Baohua Zhang, Peng Hou, Weihao Hu, et al. "A reactive power dispatch strategy with loss minimization for a DFIGbased wind farm," IEEE Transaction on Sustainable Energy, vol.7, no.3, pp: 914-923, 2016.

[26] Lijuan Chen, Zhenli Deng, and Xiaohui Xu. "Two-Stage dynamic reactive power dispatch strategy in distribution network considering the reactive power regulation of distributed generations," IEEE Transaction on Power System, vol.34, no.2, pp:1021-1032, 2019

[27] Augusto C. Rueda-Medina, John F.Franco, Marcos J.Rider, Antonio Padilha-Feltrin, Ruben Romero, “A mixed-integer linear programming approach for optimal tape, size and allocation of distributed generation radial distributed systems," Electric Power Systems Research, vol. 97, pp:133-143, 2013.

[28] Cplex solver. https://www.ibm.com/analytics/cplex-optimizer. 
[29]H. Gao, J. Liu. "Coordinated planning considering different types of DG and load in active distribution network," Proceedings of the CSEE, vol.36, no.18, pp: 4911-4922, 2016.

[30]Y. Cui, "Research and application on var compensation of the distribution network," North China Electric Power University, pp: 3, 2014.

Appendix. Test parameters of the test system (33-bus distribution network)

Table A Peak load data

\begin{tabular}{cccccc}
\hline Bus\# & $\begin{array}{c}\text { Active power } \\
\text { (MW) }\end{array}$ & $\begin{array}{c}\text { Reactive power } \\
\text { (Mvar) }\end{array}$ & Bus\# & $\begin{array}{c}\text { Active power } \\
\text { (MW) }\end{array}$ & $\begin{array}{c}\text { Reactive power } \\
\text { (Mvar) }\end{array}$ \\
\hline 1 & 0 & 0 & 18 & 0.09 & 0.04 \\
2 & 0.1 & 0.06 & 19 & 0.09 & 0.04 \\
3 & 0.09 & 0.04 & 20 & 0.09 & 0.04 \\
4 & 0.12 & 0.08 & 21 & 0.09 & 0.04 \\
5 & 0.06 & 0.03 & 22 & 0.09 & 0.04 \\
6 & 0.06 & 0.02 & 23 & 0.09 & 0.05 \\
7 & 0.2 & 0.1 & 24 & 0.42 & 0.2 \\
8 & 0.2 & 0,1 & 25 & 0.42 & 0.2 \\
9 & 0.06 & 0.02 & 26 & 0.06 & 0.025 \\
10 & 0.06 & 0.02 & 27 & 0.06 & 0.025 \\
11 & 0.045 & 0.03 & 28 & 0.06 & 0.02 \\
12 & 0.06 & 0.035 & 29 & 0.12 & 0.07 \\
13 & 0.06 & 0.035 & 30 & 0.2 & 0.6 \\
14 & 0.12 & 0.08 & 31 & 0.15 & 0.07 \\
15 & 0.06 & 0.01 & 32 & 0.21 & 0.1 \\
16 & 0.06 & 0.02 & 33 & 0.06 & 0.04 \\
17 & 0.06 & 0.02 & & & \\
\hline
\end{tabular}

Table B Line parameters

\begin{tabular}{cccccccccc}
\hline Line\# Starting bus & Ending bus Resistance $(\Omega)$ & \multicolumn{2}{c}{ Reactance $(\Omega)$} & \multicolumn{2}{l}{ Line\# Starting bus Ending bus Resistance $(\Omega)$ Reactance $(\Omega)$} \\
\hline 1 & 1 & 2 & 0.0922 & 0.047 & 19 & 19 & 20 & 1.5024 & 1.3554 \\
2 & 2 & 3 & 0.493 & 0.2511 & 20 & 20 & 21 & 0.4095 & 0.4784 \\
3 & 3 & 4 & 0.366 & 0.1864 & 21 & 21 & 22 & 0.7089 & 0.9373 \\
4 & 4 & 5 & 0.3811 & 0.1941 & 22 & 3 & 23 & 0.4512 & 0.3083 \\
5 & 5 & 6 & 0.891 & 0.707 & 23 & 23 & 24 & 0.898 & 0.7091 \\
6 & 6 & 7 & 0.1872 & 0.6188 & 24 & 24 & 25 & 0.896 & 0.7011 \\
7 & 7 & 8 & 0.7114 & 0.2351 & 25 & 6 & 26 & 0.203 & 0.1034 \\
8 & 8 & 9 & 1.03 & 0.74 & 26 & 26 & 27 & 0.2842 & 0.1447 \\
9 & 9 & 10 & 1.044 & 0.74 & 27 & 27 & 28 & 1.059 & 0.9337 \\
10 & 10 & 11 & 0.1966 & 0.065 & 28 & 28 & 29 & 0.8042 & 0.7006
\end{tabular}




\begin{tabular}{cccccccccc}
11 & 11 & 12 & 0.3744 & 0.1238 & 29 & 29 & 30 & 0.5075 & 0.2585 \\
12 & 12 & 13 & 1.468 & 1.155 & 30 & 30 & 31 & 0.9744 & 0.963 \\
13 & 13 & 14 & 0.5416 & 0.7129 & 31 & 31 & 32 & 0.3105 & 0.3619 \\
14 & 14 & 15 & 0.591 & 0.526 & 32 & 32 & 33 & 0.341 & 0.5302 \\
15 & 15 & 16 & 0.7436 & 0.545 & 33 & 8 & 21 & 2 & 2 \\
16 & 16 & 17 & 1.289 & 1.721 & 34 & 12 & 22 & 2 & 2 \\
17 & 17 & 18 & 0.732 & 0.574 & 35 & 18 & 33 & 0.5 & 0.5 \\
18 & 2 & 19 & 0.164 & 0.1565 & 36 & 25 & 29 & 0.5 & 0.5 \\
\hline
\end{tabular}

